\title{
Limited joint mobility in subjects with insulin dependent diabetes mellitus: relationship with eye and kidney complications
}

University of Colorado Health Science Center, Denver, Colorado, USA, Department of Pediatrics Satish K Garg

H Peter Chase Sandy Hoops Sherrie Harris

Department of Preventive Medicine and Biometrics Guillermo Marshall

Department of Ophthalmology William E Jackson Douglas Holmes

Correspondence to: Professor H Peter Chase, Barbara Davis Center, Box B140, University, Colorado Health 4200 East 9th Denver, Colorado 80262 USA.

Accepted 16 September 1991

Satish K Garg, H Peter Chase, Guillermo Marshall, William E Jackson, Douglas Holmes, Sandy Hoops, Sherrie Harris

\begin{abstract}
Three hundred and fifty seven subjects (178 males and 179 females) with insulin dependent diabetes mellitus were evaluated for the presence of limited joint mobility of the interphalangeal joints. Sixty six subjects (19\%) had stage 1 and 26 subjects (7\%) had stage 2 involvement of their interphalangeal joints. The presence of contractures was significantly related to mean longitudinal glycated haemoglobin $\left(\mathbf{H b A}_{1}\right)$ concentrations, duration of diabetes, age of onset, mean longitudinal cholesterol concentrations and blood pressure. Limited joint mobility was also significantly associated with early diabetic retinopathy and raised albumin excretion rates. Limited joint mobility remained a significant factor in the logistic regression model for albuminuria and grade of retinopathy when controlled for smoking, cholesterol concentrations, duration of diabetes, age, gender, and blood pressure. However, limited joint mobility was only significantly associated with diabetic retinopathy when the effect of $\mathbf{H b A}_{1}$ concentrations was included in the multivariate model.
\end{abstract}

Diabetes mellitus is the leading cause of new cases of blindness from the ages of 20-74 years $^{12}$ and the number one cause of new cases of dialysis is adults ${ }^{3}$ in the US. Poor metabolic control, ${ }^{4}$ duration of diabetes, high normal blood pressure, ${ }^{56}$ and smoking ${ }^{7}$ have been shown to be associated with early diabetic retinopathy and/or early renal damage.

Limited joint mobility has been observed with variable prevalence $(8 \cdot 4 \%-46 \%)$ in subjects with insulin dependent diabetes. ${ }^{89}$ These contractures may involve the hands, feet, and larger joints. Thickening of the skin (waxy appearance) had also been associated with limited joint mobility. ${ }^{10}$ An increased risk for diabetic microvascular complications in subjects with limited joint mobility has also been described. ${ }^{10}$ The increased prevalence of microvascular complications in subjects with limited joint mobility may be partly due to other associated factors. Previous reports have not used multivariate statistical models such as logistic regression to control for the effects of longitudinal glycated haemoglobin $\left(\mathrm{HbA}_{1}\right)$ concentrations, duration of diabetes, smoking, blood pressure levels, and other confounding variables when analysing the data for an association of limited joint mobility with early diabetic retinopathy and/or renal damage.

The purpose of this study was to evaluate the relationship of limited joint mobility with early diabetic renal and retinal damage. The influences of other factors were controlled using logistic regression models.

\section{Subjects and methods}

Three hundred and fifty seven subjects (178 males and 179 females) with insulin dependent diabetes who were at least 14 years old and who had had diabetes for at least five years were evaluated for renal and retinal changes in our eye-kidney clinic. The eye-kidney clinic began in 1986 and after their initial evaluation, subjects were asked to return at least once a year. Subjects were included in this report only if they had brought in a minimum of two overnight timed urine samples for microalbumin determinations. There were no other exclusion criteria for the study. Approximately $95 \%$ of eligible subjects from our diabetic clinic have been evaluated in the eye-kidney clinic.

During routine physical examination, each subject was asked to approximate the palmar surfaces of the interphalangeal joints of both hands with fingers extended. Normal approximation at the interphalangeal joints is usually a minimum of $180^{\circ}$. Subjects are designated by numbers of fingers with interphalangeal joint contractures as stage 0 (no limitation), stage 1 (one interphalangeal joint involved in both hands), or stage 2 (two or more interphalangeal joints involved with or without one large joint involvement).

All subjects had direct ophthalmoscopy (with pupils dilated) by at least two examiners (one ophthalmologic, and one paediatric) followed by colour retinal photographs, intravenous fluorescein angiograms and a slit lamp examination during their initial visit to the eye-kidney clinic. Subsequent visits included repeating all measurements except fluorescein angiography. Retinal findings were graded using a modified Airlie House classification of diabetic retinopathy. ${ }^{11} 12$ Subjects were classified into three groups based on the eye findings of the worst eye (grade 1: no retinal change, grade 2: microaneurysms only, and grades 3 to 6: more advanced retinopathy). The retinal specialist made the final grading with no knowledge of previous eye grades, joint contractures, glycaemic control, smoking status, blood pressure, or albumin excretion rate values, so that the grading was done in a masked fashion.

Timed overnight urine samples were collected for albumin excretion rate (AER) determinations as previously described. ${ }^{4}{ }^{13}$ Albumin concentrations were determined by radioimmunoassay 
(albumin double-antibody kit, Diagnostic Products Corporation). The subjects with or without limited joint mobility were then classified into three groups based on their overnight AER: (i) normal, less than $7 \cdot 6 \mu \mathrm{g} / \mathrm{min}$, (ii) borderline, $7 \cdot 6-30.0 \mu \mathrm{g} / \mathrm{min}$ or, (iii) abnormal, over $30 \mu \mathrm{g} / \mathrm{min}$. The accuracy of microalbumin concentrations at the lower limit was improved by routinely including a $2.5 \mathrm{mg} / 1$ standard in the samples for the standard curve. The AER detection limit by this method in our laboratory is $0.5 \mu \mathrm{g} / \mathrm{min}$, our interassay and intra-assay coefficients of variation are $1 \cdot 7-4 \cdot 4 \%$ and $3 \cdot 6-7 \cdot 4 \%$, respectively. ${ }^{13}$

Serial $\mathrm{HbA}_{1}$ values for all subjects were determined using ion exchange resin, ${ }^{4}$ (Isolab fast haemoglobin test system, Isolab Inc) with normal concentrations of $6 \cdot 3$ to $8 \cdot 2 \%$. The normal values for $\mathrm{HbA}_{1}$ have remained in this range since 1979. A mean of $7 \cdot 6$ years of $\mathrm{HbA}_{1}$ concentrations were available for each subject and for the purposes of this study a mean of all values ever recorded was used for data analyses. Serum cholesterol was measured by enzymatic assay using the Cobas autoanalyser and Beckman reagents.

Blood pressure was measured at each visit as previously described. ${ }^{7}$ Subjects were considered to have a 'high normal' systolic and/or diastolic blood pressure if levels above the 90th centile for age were detected on at least two separate clinic visits and were lower than $141 / 90 \mathrm{~mm} \mathrm{Hg}$, the level defining hypertension. All subjects signed a consent form approved by the University of Colorado Health Sciences Center human subjects commitee. Smoking was defined as previously described. ${ }^{9}$

\section{STATISTICAL METHODS}

Statistical analyses included use of $\chi^{2}$ test, Pearson correlation coefficients, and analysis of variance. When significant differences were found using analysis of variance, Dunnett's multiple $t$ test was used to compare individual groups.

Multinomial logistic regression for ordinal response variables (also called proportional odds model) was used to assess the influence of limited joint mobility on diabetic retinopathy (grade 1, 2 and 3-6) and raised AER (normal, borderline, and abnormal) with and without the presence of other factors such as age, sex, duration of diabetes, $\mathrm{HbA}_{1}$ concentrations, smoking, cholesterol concentrations, and blood pressure. A multinomial logistic regression was also used to assess the influence of the above factors on limited joint mobility.

\section{Results}

Limited joint mobility was present in $26 \%$ of the subjects (table 1). The subjects were classified into the three groups depending on the presence and stage of limited joint mobility. The mean (SEM) ages for subjects in the three groups were similar (table 1). The age ranges for the three groups, stages 0,1 , and 2 , were 14 to 30,14 to 34 , and 14 to 34 years, respectively. The mean (SEM) duration of diabetes was 10.5 $(0.2), 14 \cdot 0(0.6)$, and $14.0(0.8)$ years for subjects with stages 0,1 , and 2 , respectively $(\mathrm{p}<0.001$, analysis of variance). Sex distribution was not significantly different for subjects with or without limited joint mobility $\left(p=0.08, \chi^{2}\right.$ test), although a tendency for more males having limited joint mobility was observed (table 1). Tobacco consumption and mean systolic blood pressure were not different between the three groups. Cholesterol concentrations were significantly different $(p=0.024$, analysis of variance) for the three groups (table 1). Limited joint mobility was significantly associated with mean $\mathrm{HbA}_{\mathbf{l}}$ concentrations (table 1). Mean $\mathrm{HbA}_{1}$ concentrations were significantly higher in subjects with grade 2 limited joint mobility than subjects without limited joint mobility (Dunnett's multiple $t$ test).

Mean diastolic blood pressures were also significantly different between the three groups $(p=0.005$, analysis of variance, table 1$)$. When individual comparisons were made, only subjects with stage 2 limited joint mobility had significantly different levels from subjects without limited joint mobility. Hypertension was detected in seven subjects and was similarly distributed in the three groups. High normal blood pressure was observed in $14 \%(37 / 265)$ with stage 0 , in $14 \%(9 / 66)$ with stage 1 , and in $47 \%$ (12/26) with stage 2 limited joint mobility. A significant difference was observed in the frequency of high normal blood presssure among the three groups, especially in subjects with stage 2 limited joint mobility $\left(p=0.01, \chi^{2}\right.$ test $)$.

Using actual values the joint classification correlated significantly with the AER ( $r=0.31$, $p<0.001$; Pearson correlation coefficient). The distribution of subjects by AER categories among the three groups of limited joint mobility was statistically different $\left(p<0.002, \chi^{2}\right.$ test, table 2). Forty six percent of subjects with stage 2 limited joint mobility had AER values above $30 \mu \mathrm{g} / \mathrm{min}$ compared with $23 \%$ of subjects with stage 1 and $15 \%$ of subjects without limited joint mobility.

Using actual values, the joint classification also correlated significantly with the grade of

Table 1 Demographic data of subjects with various stages of limited joint mobility

\begin{tabular}{|c|c|c|c|c|c|c|c|c|c|}
\hline \multirow{2}{*}{$\begin{array}{l}\text { Stage of } \\
\text { limited } \\
\text { joint } \\
\text { mobility }\end{array}$} & \multirow{2}{*}{$\begin{array}{l}\text { No } \\
(\%)\end{array}$} & \multirow{2}{*}{$\begin{array}{l}\text { Mean (SEM) } \\
\text { age at } \\
\text { last visit } \\
\text { (years) }\end{array}$} & \multirow{2}{*}{$\begin{array}{l}\text { Mean (SEM) } \\
\text { duration } \\
\text { of diabetes } \\
\text { (years) }\end{array}$} & \multirow{2}{*}{$\begin{array}{l}\text { Sex } \\
(M / F)\end{array}$} & \multirow{2}{*}{$\begin{array}{l}\text { Smoking } \\
(\text { Yes/No })\end{array}$} & \multicolumn{2}{|c|}{ Mean (SEM) blood pressure ( $\mathrm{mm} \mathrm{Hg}$ ) } & \multirow{2}{*}{$\begin{array}{l}\text { Mean }(S E M) \\
H b A_{l}(\%)\end{array}$} & \multirow{2}{*}{$\begin{array}{l}\text { Mean (SEM) } \\
\text { cholesterol } \\
(\text { mmolll })\end{array}$} \\
\hline & & & & & & Systolic & Diastolic & & \\
\hline $\begin{array}{l}0 \\
1 \\
2 \\
\text { p Value }\end{array}$ & $\begin{array}{c}265(74) \\
66(19) \\
26(7)\end{array}$ & $\begin{array}{l}19.5(0.2) \\
21.0(0.5) \\
19.5(0.8) \\
>0.05 \dagger\end{array}$ & $\begin{array}{l}10 \cdot 5(0 \cdot 2) \\
14 \cdot 0(0 \cdot 6)^{*} \\
14 \cdot 0(0 \cdot 8)^{*} \\
0 \cdot 001 \dagger\end{array}$ & $\begin{array}{c}123 / 142 \\
40 / 26 \\
15 / 11 \\
>0.05 \ddagger\end{array}$ & $\begin{array}{c}201 / 64 \\
47 / 19 \\
19 / 7 \\
>0.05 \ddagger\end{array}$ & $\begin{array}{l}114.4(0.7) \\
116.3(1.3) \\
118.9(2.5) \\
>0.05 \dagger\end{array}$ & $\begin{array}{l}71.5(0.5) \\
70 \cdot 2(1.3) \\
76.7(1.6)^{*} \\
0.005 \dagger\end{array}$ & $\begin{array}{l}11.40(0.09) \\
11 \cdot 73(0.19) \\
12.47(0.82)^{*} \\
0.0014 \dagger\end{array}$ & $\begin{array}{l}4 \cdot 6(0 \cdot 05) \\
4 \cdot 7(0 \cdot 09) \\
5 \cdot 1(0 \cdot 23)^{*} \\
0 \cdot 024 \dagger\end{array}$ \\
\hline
\end{tabular}

* Significantly different when compared with value for no limited joint mobility (stage 0 ), using Dunnett's multiple $t$ test. 
Table 2 Number (\%) of subjects as classified by limited joint mobility and $A E R^{*}$

\begin{tabular}{|c|c|c|c|}
\hline \multirow[t]{2}{*}{$A E R(\mu g / \min )$} & \multicolumn{3}{|c|}{ Limited joint mobility } \\
\hline & Stage 0 & Stage 1 & Stage 2 \\
\hline $\begin{array}{l}<7 \cdot 6 \\
7 \cdot 6-30 \\
>30\end{array}$ & $\begin{array}{r}158(60) \\
67(25) \\
40(15)\end{array}$ & $\begin{array}{l}35(53) \\
16(24) \\
15(23)\end{array}$ & $\begin{array}{r}8(31) \\
6(23) \\
12(46)\end{array}$ \\
\hline Total & $265(100)$ & $66(100)$ & $26(100)$ \\
\hline
\end{tabular}

${ }^{*} \chi^{2}$ test $=0.002$

Table 3 Number (\%) of subjects as classified by limited joint mobility and grade of retinopathy

\begin{tabular}{|c|c|c|c|}
\hline \multirow[t]{2}{*}{ Eye grades } & \multicolumn{3}{|c|}{ Limited joint mobility } \\
\hline & Stage 0 & Stage 1 & Stage 2 \\
\hline $\begin{array}{l}1 \\
2 \\
3-6\end{array}$ & $\begin{array}{r}106(40) \\
98(37) \\
61(23)\end{array}$ & $\begin{array}{l}14(21) \\
17(26) \\
35(53)\end{array}$ & $\begin{array}{c}2(8) \\
9(34) \\
15(58)\end{array}$ \\
\hline Total & $265(100)$ & $66(100)$ & $26(100)$ \\
\hline
\end{tabular}

${ }^{*} \chi^{2}$ test $=0 \cdot 001$.

retinopathy ( $r=0.36, p<0.001$; Pearson correlation coefficient). The distribution of subjects by grade of retinopathy and limited joint mobility was also significantly different $\left(p<0.001, \chi^{2}\right.$ test, table 3$)$. Subjects with limited joint mobility had more advanced diabetic retinopathy as compared with subjects without limited joint mobility.

Multivariate logistic regression was used to assess the relative importance of gender, duration of diabetes, blood pressure, mean $\mathrm{HbA}_{1}$ concentrations, smoking status, and cholesterol concentrations on AER and retinal grades. When age, sex, duration of diabetes, smoking, cholesterol, and blood pressure were controlled using the logistic regression model, limited joint mobility remained significantly associated with diabetic retinopathy and AER values (as described in methods). However, when $\mathrm{HbA}_{1}$ was included in this model, limited joint mobility remained significantly associated only with diabetic retinopathy.

Using logistic regression with limited joint mobility as a response variable, the only factors having a significant effect were duration of diabetes and mean $\mathrm{HbA}_{1}$ values.

\section{Discussion}

Twenty six percent of the 357 young subjects with insulin dependent diabetes in this study demonstrated limited joint mobility. The presence of limited joint mobility was significantly associated with the duration of diabetes, with longitudinal $\mathrm{HbA}_{1}$ and cholesterol concentrations, and with diastolic blood pressure. The latter three factors were primarily associated with stage 2 joint contractures (table 1). Joint contractures of the fingers were also significantly associated with a greater likelihood of having increased AER and retinal changes. However, when longitudinal $\mathrm{HbA}_{\mathbf{1}}$ concentrations were considered in the logistic regression model, only diabetic retinal changes remained significantly associated with limited joint mobility.
The relationship between limited joint mobility, diabetic renal and retinal complications, and glucose control has previously been confusing. The initial study noting the association of limited joint mobility with microvascular disease did not attempt to differentiate between renal and retinal damage. ${ }^{8}$ It is now known that there is a relationship between longitudinal glucose control, as monitored by longitudinal $\mathrm{HbA}_{1}$ concentrations, and both the renal and the retinal complications of diabetes. ${ }^{4}$ Increased glycosylation of connective tissue has been shown in biopsy specimens of skin taken from diabetic subjects with limited joint mobility. However, several studies have not shown a relationship between $\mathrm{HbA}_{1}$ and limited joint mobility. The most likely reason for this relates to previous studies using a single $\mathrm{HbA}_{1}$ value, reflecting glucose control for only a short period. This single value may not be representative of the accumulative effect of glucose control over the many years of having diabetes. In the present study, when longitudinal $\mathrm{HbA}_{1}$ concentrations from a mean period of $7 \cdot 6$ years were studied, the limited joint mobility clearly related to the $\mathrm{HbA}_{1}$.

Another reason for the variability in reports of the relationship of limited joint mobility and diabetic renal and retinal complications and glucose control relates to inadequate statistical methods used in most previous papers. Most previous studies have not evaluated the effect of limited joint mobility on the renal and retinal complications when controlled by longitudinal $\mathrm{HbA}_{1}$ concentrations using a multivariate statistical model. When logistic regression analysis was used in the present study, limited joint mobility had no statistically significant influence on diabetic renal disease, although a significant effect on retinal disease remained. In the only other study of limited joint mobility in which logistic regression models were used (in adults with non-insulin dependent diabetes), limited joint mobility was also found to be significantly associated with retinopathy, but not with the AER. ${ }^{10}$

The main association of limited joint mobility and $\mathrm{HbA}_{1}$ concentrations was for subjects who had two or more joints involved (table 1). Studies in which different grades of involvement were not considered in the statistical analysis might have missed this association. It is our current hypothesis that when only the interphalangeal joints of the little finger are involved, a genetic aetiology may be predominant. However, when other joints are also involved, longitudinal poor glucose control is likely to be a major factor. In support of this hypothesis is the frequent finding of limited joint mobility of the interphalangeal joints of the little finger in first degree relatives of children with insulin dependent diabetes. ${ }^{9}$ However, we have never seen involvement of other joints in non-diabetic first degree relatives.

The mechanism by which limited joint mobility continues to influence retinal changes, even when the effect of longitudinal $\mathbf{H b A}_{1}$ concentrations are taken into account is unknown. It has been suggested that the initial lesion in the retina relates to loss of endothelial 
connective tissue support cells. Possibly, similar changes in connective tissue might also occur in the skin and joint capsules. The pathophysiology of diabetic retinopathy is also related to factors other than just longitudinal glucose control, such as the duration of diabetes. ${ }^{4}$ Duration of diabetes was also found to be related to the presence of limited joint mobility in the present study. Further studies are clearly needed to identify other factors influencing the development of diabetic retinal disease.

Our data suggest an association of joint contractures with the eye complications of diabetes. $\mathrm{HbA}_{1}$ was an important factor influencing joint contractures. The association of joint contractures with eye (but not kidney) changes remained when $\mathrm{HbA}_{1}$ concentrations (and other variables) were included in the multivariate model.

This study was supported in part by the Children's Diabetes Foundation at Denver and by a grant (RR-69) from the General Clinical Research Centers Program, the National Center for Research Resources, National Institutes of Health, Bethesda, Maryland.

1 Klein R, Klein BEK, Moss SE, Davis MD, DeMets DL. The Wisconsin epidemiologic study of diabetic retinopathy, II: prevalence and risk of diabetic retinopathy when age at diagnosis is less than 30 years. Arch Ophthalmol 1984;102: $520-6$.

2 Klein R, Klein BEK, Moss SE, Davis MD, DeMets DL. The Wisconsin epidemiologic study of diabetic retinopathy, III: prevalence and risk of diabetic retinopathy when age at prevalence and risk of diabetic retinopathy when age at
diagnosis is more than 30 years. Arch Ophthalmol 1984;102:

3 Health C.

stage renal disease. Administration. Research report, endstage renal disease. Baltimore: Office of Research
Demonstration, 1985. (Publication No 032741987.)

4 Chase HP, Jackson WE, Hoops SL, Cockerham RS, Archer PG, O'Brien D. Glucose control and the renal and retinal complications of insulin-dependent diabetes. $\mathscr{F} A M A 1989$; 261:1155-60.

5 Chase HP, Garg SK, Harris S, Hoops SL, Marshall G. Highnormal blood pressure and early diabetic nephropathy. Arch Intern Med 1990;150:639-41.

6 Chase HP, Garg SK, Jackson WE, et al. Blood pressure and retinopathy in type I diabetes. Ophthalmology 1990;97: 155-9.

7 Chase HP, Garg SK, Marshall G, et al. Cigarette smoking increases the risk of albuminuria among subjects with type I diabetes. $\mathcal{F A M A}$ 1991;265:614-7.

8 Rosenbloom AL, Silverstein JH, Lezotte DC, Richardson K, McCallum M. Limited joint mobility in childhood diabetes mellitus indicates increased risk for microvascular disease. N Engl f Med 1981;305:191-4.

9 Traisman HS, Traisman ES, Marr TJ, Wise J. Joint contractures in patients with juvenile diabetes and their siblings. Diabetes Care 1978;1:360-1

10 Jennings AM, Milner PC, Ward JC. Hand abnormalities are associated with the complications of diabetes in type 2 diabetes. Diabetic Med 1989;6:43-7.

11 Diabetic Retinopathy Study Research Group. Report 7: a modification of the Airlie House classification of diabetic retinopathy. Invest Ophthalmol Vis Sci 1981;21:210-26.

12 Klein BE, Davis MD, Segal P, et al. Diabetic retinopathy assessment of severity and progression. Ophthalmology 1984;91:10-7.

13 Osberg I, Chase HP, Garg SK, et al. Effects of storage time and temperature on measurement of small concentrations of albumin in urine. Clin Chem 1990;36:1428-30. 